\title{
Ekeland's variational principle in locally $p$-convex spaces and related results
}

by

\author{
J. H. QIU (Suzhou) and S. Rolewicz (Warszawa)
}

\begin{abstract}
In the framework of locally $p$-convex spaces, two versions of Ekeland's variational principle and two versions of Caristi's fixed point theorem are given. It is shown that the four results are mutually equivalent. Moreover, by using the local completeness theory, a $p$-drop theorem in locally $p$-convex spaces is proven.
\end{abstract}

1. Introduction. The variational principle discovered by Ekeland (see [6]) in 1972 is one of the most important results of nonlinear functional analysis and it has significant applications in optimization, control theory, game theory, global analysis and various other fields; see, for example, [1, $7,8,18]$. It is well known that this principle is equivalent to Caristi's fixed point theorem [2], to Daneš' drop theorem [4, 5], and to the petal theorem $[10,19]$. In the past decade, Ekeland's variational principle and some related results were extended from the Banach space setting to the topological vector space setting, in particular, to locally convex spaces (see, for example, $[3,9$, $11,17,22-24,28])$. By using the local convergence and local completeness theory on locally convex spaces (we refer to [12, p. 225] and [20, Chap. 5]), we presented general versions of Ekeland's variational principle and some related theorems in locally convex spaces (see [23], [24]). Recently [25] we introduced the notion of local completeness of locally $p$-convex spaces and established a general version of the Borwein-Preiss variational principle in locally $p$-convex spaces. It is natural to consider extensions of Ekeland's variational principle and the related results to locally complete locally $p$-convex spaces. This is the main topic of the present paper.

The paper is organized as follows. In Section 2, we recall some basic facts on $p$-convex sets, absolutely $p$-convex sets and locally complete locally

2000 Mathematics Subject Classification: 46A16, 49J40, 47H10, 49J53.

Key words and phrases: locally $p$-convex space, local completeness, Ekeland's variational principle, Caristi's fixed point theorem, Daneš' drop theorem.

Research of J. H. Qiu supported by the National Natural Science Foundation of China (10571035). 
p-convex spaces. In Section 3, using a modified version of the Bishop-Phelps lemma [21] we give two versions of Ekeland's variational principle in locally complete locally $p$-convex spaces and prove that they are indeed equivalent. In Section 4, by using the two versions of Ekeland's variational principle of Section 3, we deduce two versions of Caristi's fixed point theorem in locally $p$-convex spaces. It turns that the two versions of fixed point theorems and the two versions of Ekeland's variational principle are all equivalent to each other. Finally, in Section 5, we give a $p$-drop theorem in locally $p$-convex spaces, which extends the result in [15]. Our proof is direct and only depends on the local completeness of locally $p$-convex spaces.

2. Preliminaries and notations. In this paper we need some knowledge of $p$-convex sets and absolutely $p$-convex sets (see, for example, [13-16, 26-27]). Moreover, we need the notions of local convergence, local closedness and local completeness in locally $p$-convex spaces (for details, see [25]), which generalize the corresponding notions in locally convex spaces (see, for example, [20]).

Definition 2.1 (see $[13,14,16,27]$ ). Let $X$ be a linear space, $0<p \leq 1$ and $C \subset X$ be nonempty. If $\alpha x+\beta y \in C$ for any $x, y \in C$ and any $\alpha, \beta \geq 0$ with $\alpha^{p}+\beta^{p}=1$, then $C$ is called $p$-convex. If $C$ is in addition circled then it is called absolutely p-convex. Obviously $C$ is absolutely $p$-convex if and only if $\alpha x+\beta y \in C$ for any $x, y \in C$ and any scalars $\alpha, \beta$ such that $|\alpha|^{p}+|\beta|^{p} \leq 1$.

By induction, $C$ is $p$-convex (resp. absolutely $p$-convex) if and only if $\sum_{i=1}^{n} \alpha_{i} A \subset A$ whenever $\alpha_{i} \geq 0$ with $\sum_{i=1}^{n} \alpha_{i}^{p}=1$ (resp. whenever $\sum_{i=1}^{n}\left|\alpha_{i}\right|^{p}$ $\leq 1$ ), with $n$ running through all positive integers. It is easy to verify that arbitrary intersections of $p$-convex sets (resp. absolutely $p$-convex sets) are still $p$-convex (resp. absolutely $p$-convex). Thus for any nonempty $S \subset X$ there is a smallest $p$-convex set (resp. absolutely $p$-convex set) containing $S$, which is called the $p$-convex hull of $S$ (resp. the absolutely p-convex hull of $S$ ). We denote the $p$-convex hull of $S$ by $p-\operatorname{co}(S)$ and the absolutely $p$-convex hull of $S$ by $p$-aco $(S)$. It is easy to see that

$$
\begin{aligned}
p-\operatorname{co}(S) & =\left\{\sum_{i=1}^{n} \alpha_{i} x_{i}: n \in \mathbb{N}, x_{1}, \ldots, x_{n} \in S, \alpha_{1}, \ldots, \alpha_{n} \geq 0, \sum_{i=1}^{n} \alpha_{i}^{p}=1\right\}, \\
p-\operatorname{aco}(S) & =\left\{\sum_{i=1}^{n} \alpha_{i} x_{i}: n \in \mathbb{N}, x_{1}, \ldots, x_{n} \in S, \sum_{i=1}^{n}\left|\alpha_{i}\right|^{p} \leq 1\right\} .
\end{aligned}
$$

Let $x \in X$ and $B \subset X$ be nonempty. We denote $p$-co $(\{x\} \cup B)$ by $D_{p}(x, B)$, and $p$-aco $(\{x\} \cup B)$ by $\Gamma_{p}(x, B)$. If $B$ is $p$-convex, we call $D_{p}(x, B)$ the $p$-drop determined by $x$ and $B$. 
Remark 2.1. Assume that $0<p<1$ and $C$ is a $p$-convex set in a linear space $X$. Then $\alpha x \in C$ for any $x \in C$ and any $\alpha$ such that $2^{1-1 / p} \leq \alpha \leq 1$. Hence $\alpha x \in C$ for any $x \in C$ and any $0<\alpha \leq 1$. Thus either 0 belongs to $C$ or it is an algebraic boundary point of $C$ (see [14, p. 177]). If the $p$-convex set $C$ is algebraic closed or locally closed, then clearly $0 \in C$. Hence for any nonempty subset $S$ of $X, p-\operatorname{co}(S)$ can also be written as

$$
p-\operatorname{co}(S)=\left\{\sum_{i=1}^{n} \alpha_{i} x_{i}: n \in \mathbb{N}, x_{1}, \ldots, x_{n} \in S, \alpha_{1}, \ldots, \alpha_{n} \geq 0,0<\sum_{i=1}^{n} \alpha_{i}^{p} \leq 1\right\} .
$$

Besides, we remark that if $C$ is a convex set (i.e. $p=1$ ), we cannot deduce $\alpha x \in C$ from $x \in C$ and $0<\alpha<1$.

Remark 2.2. From Remark 2.1 we know that for any $p$-convex set $C$ $(0<p<1)$, we can define its gauge as follows:

$q_{C}(x)= \begin{cases}\inf \left\{t>0: x \in t^{1 / p} C\right\} & \text { if there exists } t>0 \text { such that } x \in t^{1 / p} C, \\ \infty & \text { else. }\end{cases}$

Clearly, for any $x, y \in X$ and any $\alpha>0$, we have $q_{C}(x+y) \leq q_{C}(x)+q_{C}(y)$ and $q_{C}(\alpha x)=\alpha^{p} q_{C}(x)$. Moreover, if $C$ is absorbing, then $q_{C}(0)=0$ and $0 \leq q_{C}(x)<\infty$ for any $x \in X$.

For any convex set $C$ containing 0 , we can define its gauge as follows:

$$
q_{C}(x)= \begin{cases}\inf \{t>0: x \in t C\} & \text { if there exists } t>0 \text { such that } x \in t C, \\ \infty & \text { else. }\end{cases}
$$

Clearly, for any $x, y \in C$ and any $\alpha \geq 0$, we have $q_{C}(x+y) \leq q_{C}(x)+q_{C}(y)$ and $q_{C}(\alpha x)=\alpha q_{C}(x)$. Moreover, if $C$ is absorbing, then $0 \leq q_{C}(x)<\infty$ for any $x \in X$.

In general, a function $q: X \rightarrow \mathbb{R} \cup\{+\infty\}$ is called subadditive if $q(x+y)$ $\leq q(x)+q(y)$ for any $x, y \in X$, and positive p-homogeneous if $q(\alpha x)=$ $\alpha^{p} q(x)$ for any $x \in X$ and $\alpha \geq 0$ (when $p=1$, we call it briefly positive homogeneous). Obviously, the gauge of a $p$-convex set $(0<p \leq 1)$ containing 0 is a subadditive, positive $p$-homogeneous function.

Now we turn to the discussion of locally $p$-convex spaces. A Hausdorff topological linear space (briefly, a topological linear space) is called locally $p$-convex $(0<p \leq 1)$ if there is a basis $\mathcal{U}$ of 0 -neighborhoods consisting of absolutely $p$-convex sets. It is easy to see that a topological linear space $X$ is locally $p$-convex if and only if there is a family $\left\{\|\|_{\lambda}\right\}_{\lambda \in \Lambda}$ of $p$-homogeneous F-pseudonorms which determines the topology of $X$ (see, for example, [27]). In particular, a locally 1-convex space is called locally convex.

Definition 2.2 (see [25]). Let $X$ be a locally $p$-convex space. A subset $B$ of $X$ is called a $p$-disc if it is bounded and absolutely $p$-convex. Let $E_{B}$ 
denote the linear span of $B, \operatorname{span}[B]$, endowed with the topology determined by the gauge $q_{B}$. If $E_{B}$ is complete, then the $p$-disc $B$ is called self-complete. A sequence $\left(x_{n}\right)$ in $X$ is called a locally Cauchy sequence if there is a $p$-disc $B$ in $X$ such that $\left(x_{n}\right)$ is a Cauchy sequence in $E_{B}$. A sequence $\left(x_{n}\right)$ in $X$ is said to be locally convergent to a point $x_{0}$ if there is a $p$-disc $B$ such that $x_{n} \rightarrow x_{0}$ in $E_{B}$. Clearly, $\left(x_{n}\right)$ is locally convergent to $x_{0}$ if and only if $\left(x_{n}-x_{0}\right)$ is locally convergent to 0 . A point $x_{0}$ is called a locally limit point of a set $A$ in $X$ if there is a sequence $\left(x_{n}\right)$ in $A$ locally convergent to $x_{0}$. The set $A$ is called locally closed if every locally limit point of $A$ belongs to $A$.

Lemma 2.1 (see [25]). Let $X$ be a locally p-convex space. Then the following three statements are equivalent:

(i) For each bounded set $A$ in $X$ there is a self-complete $p$-disc $B$ such that $A \subset B$.

(ii) Every locally Cauchy sequence in $X$ is locally convergent.

(iii) Every closed p-disc is self-complete.

Definition 2.3 (see [25]). A locally $p$-convex space $X$ is called locally complete if one of the three equivalent statements in Lemma 2.1 is satisfied. A subset $A$ of a locally $p$-convex space is called locally complete if every locally Cauchy sequence in $A$ is locally convergent to a point in $A$.

Obviously, every locally complete set is locally closed and every locally closed subset of a locally complete space is locally complete.

Let $X$ be a locally $p$-convex space and let $f: X \rightarrow \mathbb{R} \cup\{+\infty\}$. The effective domain of $f$ is $\operatorname{dom} f=\{x \in X: f(x)<\infty\}$. If $\operatorname{dom} f \neq \emptyset$, we say that $f$ is proper. As in [23], we introduce the notion of locally lower semicontinuous function as follows.

Definition 2.4. Let $X$ be a locally $p$-convex space and $f: X \rightarrow \mathbb{R} \cup$ $\{+\infty\}$ be a proper function. If the set $\{x \in X: f(x) \leq r\}$ is locally closed in $X$ for every $r \in \mathbb{R}$, then $f$ is called locally lower semicontinuous.

Concerning the relationship between locally lower semicontinuous functions, sequentially lower semicontinuous functions and lower semicontinuous functions, we refer to [23]. In the next section we shall consider some extensions of Ekeland's variational principle concerning locally lower semicontinuous functions in the setting of locally $p$-convex spaces.

\section{Ekeland's variational principle in locally $p$-convex spaces.} First we give a modified version of the Bishop-Phelps lemma.

Lemma 3.1. Let $X$ be a locally complete locally $p$-convex space and $q$ : $X \rightarrow \mathbb{R}^{+} \cup\{+\infty\}$ be a locally lower semicontinuous, positive $p$-homogeneous, subadditive proper function such that $B:=\{x \in X: q(x) \leq 1\}$ is bounded. 
If a nonempty set $A \subset X \times \mathbb{R}$ is locally closed and $\inf \{r:(x, r) \in A\}=0$, then for any $\beta>0$ and any $\left(x_{0}, r_{0}\right) \in A$, there exists

$$
(x, r) \in A \cap\left(K_{\beta}+\left(x_{0}, r_{0}\right)\right)
$$

such that

$$
\{(x, r)\}=A \cap\left(K_{\beta}+(x, r)\right),
$$

where $K_{\beta}:=\{(x, r) \in X \times R: \beta q(x) \leq-r\}$.

Proof. It is easy to verify that $(0,0) \in K_{\beta}, K_{\beta}+K_{\beta} \subset K_{\beta}$ and $K_{\beta}$ is locally closed in $X \times \mathbb{R}$. Define $h: X \times \mathbb{R} \rightarrow \mathbb{R}$ as follows:

$$
h(x, r)=r, \quad \forall(x, r) \in X \times \mathbb{R} .
$$

Put $A_{0}:=A \cap\left(K_{\beta}+\left(x_{0}, r_{0}\right)\right)$. Then

$$
0=\inf h(A) \leq \inf h\left(A_{0}\right) \leq r_{0}<\infty .
$$

There exists $\left(x_{1}, r_{1}\right) \in A_{0}$ such that

$$
r_{1}<\inf h\left(A_{0}\right)+1 .
$$

Put $A_{1}:=A \cap\left(K_{\beta}+\left(x_{1}, r_{1}\right)\right)$. Then

$$
A_{1} \subset A \cap\left(K_{\beta}+K_{\beta}+\left(x_{0}, r_{0}\right)\right) \subset A \cap\left(K_{\beta}+\left(x_{0}, r_{0}\right)\right)=A_{0} .
$$

Clearly,

$$
0 \leq \inf h\left(A_{0}\right) \leq \inf h\left(A_{1}\right) \leq r_{1}<\infty .
$$

Hence there exists $\left(x_{2}, r_{2}\right) \in A_{1}$ such that

$$
r_{2}<\inf h\left(A_{1}\right)+1 / 2 .
$$

Repeating this process we obtain a sequence $\left\{\left(x_{n}, r_{n}\right)\right\}_{n \in \mathbb{N}}$ such that $\left(x_{n+1}, r_{n+1}\right) \in A_{n}=A \cap\left(K_{\beta}+\left(x_{n}, r_{n}\right)\right)$ and $r_{n+1}<\inf h\left(A_{n}\right)+\frac{1}{n+1}$.

Clearly, for any $m>n>1$,

$$
\begin{aligned}
\beta q\left(x_{m}-x_{n}\right) & \leq r_{n}-r_{m}<\inf h\left(A_{n-1}\right)+\frac{1}{n}-r_{m} \\
& \leq \inf h\left(A_{m-1}\right)+\frac{1}{n}-r_{m} \leq \frac{1}{n},
\end{aligned}
$$

so $\left\{x_{n}\right\}_{n \in \mathbb{N}}$ is a locally Cauchy sequence in $X$, and hence is locally convergent to some $x \in X$. Also, $\left\{r_{n}\right\}$ is convergent to some $r \in \mathbb{R}$. Since $A_{n}=A \cap\left(K_{\beta}+\left(x_{n}, r_{n}\right)\right)$ is locally closed, $\left(x_{m}, r_{m}\right) \in A_{n}$ for every $m>n$ and $\left\{\left(x_{m}, r_{m}\right)\right\}_{m>n}$ is locally convergent to $(x, r)$, we have $(x, r) \in A_{n}$ and hence $(x, r) \in \bigcap_{n=0}^{\infty} A_{n}$. In particular,

$$
(x, r) \in A_{0}=A \cap\left(K_{\beta}+\left(x_{0}, r_{0}\right)\right) .
$$

Next we show that $\{(x, r)\}=A \cap\left(K_{\beta}+(x, r)\right)$. Indeed, if $(y, s) \in A \cap\left(K_{\beta}+\right.$ $(x, r))$, then for every $n$,

$$
(y, s) \in A \cap\left(K_{\beta}+K_{\beta}+\left(x_{n}, r_{n}\right)\right) \subset A \cap\left(K_{\beta}+\left(x_{n}, r_{n}\right)\right)=A_{n} .
$$


Thus $\left(y-x_{n}, s-r_{n}\right) \in K_{\beta}$ and hence

$$
\beta q\left(y-x_{n}\right) \leq r_{n}-s<\inf h\left(A_{n-1}\right)+\frac{1}{n}-s \leq \inf h\left(A_{n}\right)+\frac{1}{n}-s \leq \frac{1}{n} .
$$

Therefore $\left\{x_{n}\right\}$ is locally convergent to $y$ and $\left\{r_{n}\right\}$ is convergent to $s$. By the uniqueness of limits, we have $y=x$ and $s=r$, as desired.

TheOREM 3.1. Let $X$ be a locally complete locally $p$-convex space and $q$ : $X \rightarrow \mathbb{R}^{+} \cup\{+\infty\}$ be a locally lower semicontinuous, positive $p$-homogeneous, subadditive proper function such that $B:=\{x \in X: q(x) \leq 1\}$ is bounded. Let $f: X \rightarrow \mathbb{R} \cup\{+\infty\}$ be a locally lower semicontinuous, bounded from below, proper function and let $x_{0} \in \operatorname{dom} f$. Then for any $\beta>0$, there exists $z \in X$ such that

(i) $f(z)+\beta q\left(z-x_{0}\right) \leq f\left(x_{0}\right)$;

(ii) for any $x \neq z, f(z)<f(x)+\beta q(x-z)$.

Proof. We may assume that $\inf f(X)=0$. Put $A:=\{(x, r) \in X \times \mathbb{R}$ : $f(x) \leq r\}$. Then $\inf \{r:(x, r) \in A\}=0$. Clearly $\left(x_{0}, f\left(x_{0}\right)\right) \in A$. By Lemma 3.1, there exists

$$
(z, r) \in A \cap\left(K_{\beta}+\left(x_{0}, f\left(x_{0}\right)\right)\right)
$$

such that

$$
\{(z, r)\}=A \cap\left(K_{\beta}+(z, r)\right) .
$$

From (1), we have $f(z) \leq r$ and

$$
\beta q\left(z-x_{0}\right) \leq f\left(x_{0}\right)-r \leq f\left(x_{0}\right)-f(z),
$$

proving (i).

Next we show that $f(z)=r$. If not, we have $f(z)<r$. Clearly $(z, f(z)) \in$ $A$ and by (2),

$$
(z, f(z)) \notin K_{\beta}+(z, r),
$$

which implies that

$$
(0, f(z)-r) \notin K_{\beta}, \quad \text { i.e. } \quad 0=\beta q(0)>r-f(z),
$$

contradicting $f(z)<r$. Thus $r=f(z)$ and hence (2) becomes

$$
\{(z, f(z))\}=A \cap\left(K_{\beta}+(z, f(z))\right) \text {. }
$$

If $x \in X$ and $f(x)=\infty$, then certainly (ii) holds. Let $x \in \operatorname{dom} f$ and $x \neq z$. Then by (3) we have

$$
(x, f(x)) \notin K_{\beta}+(z, f(z)),
$$

that is, $(x-z, f(x)-f(z)) \notin K_{\beta}$, or equivalently, $\beta q(x-z)>f(z)-f(x)$.

Lemma 3.2. Let $X$ be a locally p-convex space with the topology generated by a family $\left\{\|\|_{\lambda}\right\}_{\lambda \in \Lambda}$ of p-homogeneous F-pseudonorms and $\left\{\alpha_{\lambda}\right\}_{\lambda \in \Lambda}$ be a family of positive real numbers. Then $B:=\bigcap_{\lambda \in \Lambda}\left\{x \in X: \alpha_{\lambda}\|x\|_{\lambda} \leq 1\right\}$ is a 
closed, bounded, absolutely $p$-convex set in $X$, and $q_{B}(x)=\sup _{\lambda \in \Lambda} \alpha_{\lambda}\|x\|_{\lambda}$ for any $x \in X$, where

$q_{B}(x)= \begin{cases}\inf \left\{t>0: x \in t^{1 / p} B\right\} & \text { if there exists } t>0 \text { such that } x \in t^{1 / p} B \\ \infty & \text { else. }\end{cases}$

Proof. It is easy to verify that $B$ is closed, bounded, and absolutely $p$-convex. For any $x \in X$ and $\varepsilon>0$, by the definition of $q_{B}$ we have

which means that

$$
\frac{x}{\left(q_{B}(x)+\varepsilon\right)^{1 / p}} \in B
$$

Hence

$$
\alpha_{\lambda}\left\|\frac{x}{\left(q_{B}(x)+\varepsilon\right)^{1 / p}}\right\|_{\lambda} \leq 1, \quad \forall \lambda \in \Lambda .
$$

This implies that

$$
\frac{\alpha_{\lambda}}{q_{B}(x)+\varepsilon}\|x\|_{\lambda} \leq 1, \quad \forall \lambda \in \Lambda \text {. }
$$

$$
\sup _{\lambda \in \Lambda} \alpha_{\lambda}\|x\|_{\lambda} \leq q_{B}(x) .
$$

Suppose that there exist $x_{0} \in X$ and $\varepsilon>0$ such that

$$
\sup _{\lambda \in \Lambda} \alpha_{\lambda}\left\|x_{0}\right\|_{\lambda}<q_{B}\left(x_{0}\right)-\varepsilon .
$$

Then

$$
\sup _{\lambda \in \Lambda} \alpha_{\lambda}\left\|\frac{x_{0}}{\left(q_{B}\left(x_{0}\right)-\varepsilon\right)^{1 / p}}\right\|_{\lambda}=\sup _{\lambda \in \Lambda} \frac{\alpha_{\lambda}\left\|x_{0}\right\|_{\lambda}}{q_{B}\left(x_{0}\right)-\varepsilon}<1 .
$$

Hence

$$
\frac{x_{0}}{\left(q_{B}\left(x_{0}\right)-\varepsilon\right)^{1 / p}} \in B, \quad \text { i.e., } \quad x_{0} \in\left(q_{B}\left(x_{0}\right)-\varepsilon\right)^{1 / p} B .
$$

But this contradicts the definition of $q_{B}$. Therefore

$$
q_{B}(x)=\sup _{\lambda \in \Lambda} \alpha_{\lambda}\|x\|_{\lambda}, \quad \forall x \in X .
$$

TheOrem 3.2. Let $X$ be a locally complete locally $p$-convex space with the topology generated by a family $\left\{\|\|_{\lambda}\right\}_{\lambda \in \Lambda}$ of p-homogeneous F-pseudonorms and $\left\{\alpha_{\lambda}\right\}_{\lambda \in \Lambda}$ be a family of positive real numbers. Let $f: X \rightarrow \mathbb{R} \cup\{+\infty\}$ be a locally lower semicontinuous, bounded from below, proper function and let $x_{0} \in \operatorname{dom} f$. Then for any $\beta>0$, there exists $z \in X$ such that

(i) $f(z)+\beta \sup _{\lambda \in \Lambda} \alpha_{\lambda}\left\|z-x_{0}\right\|_{\lambda} \leq f\left(x_{0}\right)$;

(ii) for any $x \neq z, f(z)<f(x)+\beta \sup _{\lambda \in \Lambda} \alpha_{\lambda}\|x-z\|_{\lambda}$.

Proof. Put $B:=\bigcap_{\lambda \in \Lambda}\left\{x \in X: \alpha_{\lambda}\|x\|_{\lambda} \leq 1\right\}$. By Lemma 3.2,

$$
q_{B}(x)=\sup _{\lambda \in \Lambda} \alpha_{\lambda}\|x\|_{\lambda}, \quad \forall x \in X .
$$

Combining (4) and Theorem 3.1 completes the proof. 
Since a complete $p$-norm space is a locally complete locally $p$-convex space whose topology is generated by a single $p$-norm, from Theorem 3.2 we have the following:

Corollary 3.1. Let $(X,\|\|)$ be a complete p-normed space, $f:(X,\|\|)$ $\rightarrow \mathbb{R} \cup\{+\infty\}$ be a lower semicontinuous, bounded from below, proper function and $x_{0} \in \operatorname{dom} f$. Then for any $\beta>0$, there exists $z \in X$ such that

(i) $f(z)+\beta\left\|z-x_{0}\right\| \leq f\left(x_{0}\right)$;

(ii) for any $x \neq z, f(z)<f(x)+\beta\|x-z\|$.

TheOrem 3.3. Theorems 3.1 and 3.2 are mutually equivalent.

Proof. We only need to prove that Theorem 3.2 implies Theorem 3.1. In fact, we shall prove that Corollary 3.1 implies Theorem 3.1. Let $X, q, B$ and $f$ be as in Theorem 3.1 and let $x_{0} \in \operatorname{dom} f$. We denote by $T$ the closure of $\Gamma_{p}\left(x_{0}, B\right)$. Since $X$ is a locally complete locally $p$-convex space, by Lemma 2.1 and Definition 2.3 we know that $T$ is a self-complete $p$-disc, i.e., $\left(X_{T},\|\|_{T}\right)$ is a complete $p$-normed space. Here $X_{T}:=\operatorname{span}[T]$ and for any $x \in X_{T}$, $\|x\|_{T}:=\inf \left\{t>0: x \in t^{1 / p} T\right\}$. Put

$$
C:=\left\{x \in X_{T}: f(x)+\beta q\left(x-x_{0}\right) \leq f\left(x_{0}\right)\right\} .
$$

Then $C$ is closed in $\left(X_{T},\|\|_{T}\right)$ since $f$ and $q$ are locally lower semicontinuous. Define a function $g$ on $X_{T}$ as follows:

$$
g(x)= \begin{cases}f(x) & \text { if } x \in C, \\ \infty & \text { if } x \in X_{T} \backslash C .\end{cases}
$$

Then $g$ is a lower semicontinuous, bounded from below, proper function on $\left(X_{T},\|\|_{T}\right)$ and $x_{0} \in \operatorname{dom} g$. Now applying Corollary 3.1 to the complete $p$-normed space $\left(X_{T},\|\|_{T}\right)$ and the function $g$, we conclude that there exists $z \in X_{T}$ such that

(i) $g(z)+\beta\left\|z-x_{0}\right\|_{T} \leq g\left(x_{0}\right)=f\left(x_{0}\right)$;

(ii) for any $x \in X_{T}$ and $x \neq z$,

$$
g(z)<g(x)+\beta\|x-z\|_{T} .
$$

From (i), we know that $g(z)<\infty$ and hence $z \in C$, that is,

$$
f(z)+\beta q\left(z-x_{0}\right) \leq f\left(x_{0}\right) .
$$

Thus (i) in Theorem 3.1 holds. Next we show that (ii) in Theorem 3.1 holds in each of the following three possible cases.

Case 1 . Let $x \neq z$ and $x \in C$. Then by (5) we have

$$
f(z)<f(x)+\beta\|x-z\|_{T} .
$$

Since $T \supset B$ and \|\|$_{T}$ is the gauge of $T$, and clearly $q$ is the gauge of $B$, we have $\|\cdot\|_{T} \leq q(\cdot)$. Combining this with $(7)$, we conclude that $f(z)<$ $f(x)+\beta q(x-z)$. 
CASE 2. Let $x \neq z$ and $x \in X_{T} \backslash C$. From the definition of $C$, we have

$$
f(x)+\beta q\left(x-x_{0}\right)>f\left(x_{0}\right) .
$$

Combining this with (6), we have

$$
\begin{aligned}
f(z)+\beta q\left(z-x_{0}\right) & \leq f\left(x_{0}\right)<f(x)+\beta q\left(x-x_{0}\right) \\
& \leq f(x)+\beta q(x-z)+\beta q\left(z-x_{0}\right) .
\end{aligned}
$$

By (6), we know that $\beta q\left(z-x_{0}\right)<\infty$, so (8) yields $f(z)<f(x)+\beta q(x-z)$.

CASE 3. Let $x \neq z$ and $x \notin X_{T}$. Since $z \in X_{T}$, we have $x-z \notin X_{T}$. Thus $x-z \notin t^{1 / p} B$ for any $t>0$, so $q(x-z)=\infty$ and obviously $f(z)<$ $f(x)+\beta q(x-z)$.

Obviously Theorem 3.1 is an extension of [11, Theorem 2] and [24, Corollary 3.2]. And Theorem 3.2 is an extension of [11, Theorem 3] and [24, Corollary 3.1]. In fact, by slightly modifying Lemma 3.1 we can obtain the following extensions of [24, Theorems 3.2 and 3.1].

Theorem 3.1'. Let $X$ be a locally $p$-convex space and $q: X \rightarrow \mathbb{R}^{+} \cup$ $\{+\infty\}$ be a locally lower semicontinuous, positive $p$-homogeneous, subadditive proper function such that $B:=\{x \in X: q(x) \leq 1\}$ is bounded. Let $f: X \rightarrow$ $\mathbb{R} \cup\{+\infty\}$ be a locally lower semicontinuous, bounded from below, proper function and let $x_{0} \in \operatorname{dom} f$. If $\left\{x \in X: f(x) \leq f\left(x_{0}\right)\right\}$ or $B$ is locally complete, then for any $\beta>0$, there exists $z \in X$ such that

(i) $f(z)+\beta q\left(z-x_{0}\right) \leq f\left(x_{0}\right)$;

(ii) for any $x \neq z, f(z)<f(x)+\beta q(x-z)$.

TheOREM 3.2'. Let $X$ be a locally $p$-convex space with the topology generated by a family $\left\{\|\|_{\lambda}\right\}_{\lambda \in \Lambda}$ of p-homogeneous F-pseudonorms and $\left\{\alpha_{\lambda}\right\}_{\lambda \in \Lambda}$ be a family of positive real numbers. Let $f: X \rightarrow \mathbb{R} \cup\{+\infty\}$ be a locally lower semicontinuous, bounded from below, proper function and let $x_{0} \in \operatorname{dom} f$. If $\left\{x \in X: f(x) \leq f\left(x_{0}\right)\right\}$ or $\bigcap_{\lambda \in \Lambda}\left\{x \in X: \alpha_{\lambda}\|x\|_{\lambda} \leq 1\right\}$ is locally complete, then for any $\beta>0$, there exists $z \in X$ such that

(i) $f(z)+\beta \sup _{\lambda \in \Lambda} \alpha_{\lambda}\left\|z-x_{0}\right\|_{\lambda} \leq f\left(x_{0}\right)$;

(ii) for any $x \neq z, f(z)<f(x)+\beta \sup _{\lambda \in \Lambda} \alpha_{\lambda}\|x-z\|_{\lambda}$.

\section{Equivalence of Ekeland's variational principle and Caristi's} fixed point theorem. By using Theorems 3.1 and 3.2, we can deduce the following two versions of Caristi's fixed point theorem in locally $p$-convex spaces.

Theorem 4.1 (Extended Caristi's fixed point theorem). Let $X$ be a locally complete locally $p$-convex space and $q: X \rightarrow \mathbb{R}^{+} \cup\{+\infty\}$ be a locally lower semicontinuous, positive p-homogeneous, subadditive, proper function such that $\{x \in X: q(x) \leq 1\}$ is bounded. Let $f: X \rightarrow \mathbb{R} \cup\{+\infty\}$ be a locally 
lower semicontinuous, bounded from below, proper function. If $T: X \rightarrow 2^{X}$ has the property that for any $x \in X$ and any $y \in T x$,

$$
\beta q(y-x)+f(y) \leq f(x), \quad \text { where } \beta>0 \text { is a constant, }
$$

then for any $x_{0} \in \operatorname{dom} f$, there exists $z \in\left(T x_{0}\right)^{\sim}$ such that $T z=\{z\}$, where $\left(T x_{0}\right)^{\sim}:=\left\{y \in X: \beta q\left(y-x_{0}\right)+f(y) \leq f\left(x_{0}\right)\right\}$.

Proof. By Theorem 3.1, there exists $z \in X$ such that

(i) $f(z)+\beta q\left(z-x_{0}\right) \leq f\left(x_{0}\right)$;

(ii) for any $x \neq z, f(z)<f(x)+\beta q(x-z)$.

From (i) we know that $z \in\left(T x_{0}\right) \sim$. We show that $T z=\{z\}$. If not, there exists $y \in T z$ and $y \neq z$. From (ii), we have

$$
f(z)<f(y)+\beta q(y-z) .
$$

On the other hand, as $y \in T z$, by the hypothesis on $T$ we have $\beta q(y-z)+f(y)$ $\leq f(z)$, which contradicts $(9)$.

Similarly we can deduce the following version of Caristi's fixed point theorem from Theorem 3.2.

Theorem 4.2 (Extended Caristi's fixed point theorem). Let $X$ be a locally complete locally $p$-convex space with the topology generated by a family $\left\{\|\|_{\lambda}\right\}_{\lambda \in \Lambda}$ of p-homogeneous F-pseudonorms, $\left\{\alpha_{\lambda}\right\}_{\lambda \in \Lambda}$ be a family of positive real numbers and let $f: X \rightarrow \mathbb{R} \cup\{+\infty\}$ be a locally lower semicontinuous, bounded from below, proper function. If $T: X \rightarrow 2^{X}$ has the property that for any $x \in X$ and any $y \in T x$,

$$
\alpha_{\lambda}\|x-y\|_{\lambda}+f(y) \leq f(x), \quad \forall \lambda \in \Lambda,
$$

then for any $x_{0} \in \operatorname{dom} f$, there exists $z \in\left(T x_{0}\right) \sim$ such that $T z=\{z\}$, where $\left(T x_{0}\right)^{\sim}:=\left\{y \in X: \alpha_{\lambda}\left\|x_{0}-y\right\|_{\lambda}+f(y) \leq f\left(x_{0}\right), \forall \lambda \in \Lambda\right\}$.

In fact, the two versions of Caristi's fixed point theorem and the two versions of Ekeland's variational principle of Section 3 are equivalent to one another.

TheOREM 4.3. Theorems 3.1, 3.2, 4.1 and 4.2 are equivalent to one another.

Proof. We only need to prove that Theorem 4.1 implies Theorem 3.1 and that Theorem 4.2 implies Theorem 3.2. Here we only give the proof of the former implication; the other proof is similar. Let $X, q, B$ and $f$ be as in Theorem 3.1 and let $x_{0} \in \operatorname{dom} f$. Define $T: X \rightarrow 2^{X}$ by

$$
T x:=\{y \in X: \beta q(y-x)+f(y) \leq f(x)\} .
$$

Obviously $x \in T x$ and $T$ satisfies the hypothesis of Theorem 4.1. Hence there exists $z \in\left(T x_{0}\right)^{\sim}=\left\{y \in X: \beta q\left(y-x_{0}\right)+f(y) \leq f\left(x_{0}\right)\right\}$ such 
that $T z=\{z\}$. Thus $\beta q\left(z-x_{0}\right)+f(z) \leq f\left(x_{0}\right)$, i.e., Theorem 3.1(i) holds. Moreover, for any $x \neq z$, we have $x \notin T z$, i.e.,

$$
\beta q(x-z)+f(x)>f(z) .
$$

Thus Theorem 3.1(ii) holds.

5. A $p$-drop theorem in locally $p$-convex spaces $(0<p<1)$. In order to obtain a general $p$-drop theorem in locally $p$-convex spaces, we need the following lemmas.

Lemma 5.1 (see [15]). Let $X$ be a linear space and let $C \subset X$ be $p$-convex. Then

$$
D_{p}(x, C)=\left\{\alpha x+\beta y: y \in C, \alpha, \beta \in[0,1], \alpha^{p}+\beta^{p}=1\right\} .
$$

Proof. Fix $z \in D_{p}(x, C)$. Then $z=\alpha_{0} x+\sum_{i=1}^{n} \alpha_{i} y_{i}$, where $y_{1}, \ldots, y_{n}$ $\in C$ and $\alpha_{0}, \alpha_{1}, \ldots, \alpha_{n} \in[0,1]$ with $\sum_{i=0}^{n} \alpha_{i}^{p}=1$.

If $\alpha_{0}=1$, then clearly $z=x \in\left\{\alpha x+\beta y: y \in C, \alpha, \beta \in[0,1], \alpha^{p}+\beta^{p}=1\right\}$. If $0 \leq \alpha_{0}<1$, then

$$
z=\alpha_{0} x+\left(\sum_{i=1}^{n} \alpha_{i}^{p}\right)^{1 / p} \sum_{i=1}^{n} \frac{\alpha_{i}}{\left(\sum_{j=1}^{n} \alpha_{j}^{p}\right)^{1 / p}} y_{i} .
$$

Observe that

$$
\sum_{i=1}^{n}\left(\frac{\alpha_{i}}{\left(\sum_{j=1}^{n} \alpha_{j}^{p}\right)^{1 / p}}\right)^{p}=1, \quad y_{i} \in C \quad \text { and } \quad C \text { is } p \text {-convex }
$$

so we have

$$
\sum_{i=1}^{n} \frac{\alpha_{i}}{\left(\sum_{j=1}^{n} \alpha_{j}^{p}\right)^{1 / p}} y_{i} \in C .
$$

Since $\alpha_{0}^{p}+\left[\left(\sum_{i=1}^{n} \alpha_{i}^{p}\right)^{1 / p}\right]^{p}=1$, the proof is complete.

Lemma 5.2. Let $C$ be a p-convex set and $a \in D_{p}(x, C)$. Then $D_{p}(a, C) \subset$ $D_{p}(x, C)$.

Proof. For any $z \in D_{p}(a, C)$, by Lemma 5.1 we can write

$$
z=\alpha a+\beta y, \quad \text { where } y \in C, \alpha, \beta \in[0,1], \alpha^{p}+\beta^{p}=1 .
$$

Since $a \in D_{p}(x, C)$, we have

$$
a=\lambda x+\mu y^{\prime}, \quad \text { where } \quad y^{\prime} \in C, \lambda, \mu \in[0,1], \lambda^{p}+\mu^{p}=1 .
$$

Thus

$$
\begin{aligned}
z & =\alpha a+\beta y=\alpha\left(\lambda x+\mu y^{\prime}\right)+\beta y=\alpha \lambda x+\alpha \mu y^{\prime}+\beta y \\
& =\alpha \lambda x+\left((\alpha \mu)^{p}+\beta^{p}\right)^{1 / p} \cdot \frac{\alpha \mu y^{\prime}+\beta y}{\left((\alpha \mu)^{p}+\beta^{p}\right)^{1 / p}} .
\end{aligned}
$$


Since $C$ is $p$-convex and $y, y^{\prime} \in C$, we have

$$
\frac{\alpha \mu y^{\prime}+\beta y}{\left((\alpha \mu)^{p}+\beta^{p}\right)^{1 / p}} \in C .
$$

To complete the proof, it suffices to observe that

$$
(\alpha \lambda)^{p}+(\alpha \mu)^{p}+\beta^{p}=\alpha^{p}\left(\lambda^{p}+\mu^{p}\right)+\beta^{p}=\alpha^{p}+\beta^{p}=1 .
$$

In the following we always assume that $X$ is a locally $p$-convex space.

Lemma 5.3. Let $B \subset X$ be a locally closed, bounded, $p$-convex set, and let $x_{0} \in X$. Then $D_{p}\left(x_{0}, B\right)$ is also locally closed and bounded.

Proof. Since $X$ has a 0-neighborhood base consisting of absolutely $p$ convex sets, the absolutely $p$-convex hull of the bounded set $\left\{x_{0}\right\} \cup B$, i.e. $\Gamma_{p}\left(x_{0}, B\right)$, is bounded, and hence so is $D_{p}\left(x_{0}, B\right) \subset \Gamma_{p}\left(x_{0}, B\right)$.

Now we prove that $D_{p}\left(x_{0}, B\right)$ is locally closed. Let $\left(a_{i}\right) \subset D_{p}\left(x_{0}, B\right)$ be locally convergent to $a_{0}$, that is, there is a bounded $p$-disc $A$ such that $a_{i} \rightarrow a_{0}$ in $E_{A}$. We may assume that $A \supset \Gamma_{p}\left(x_{0}, B\right) \supset D_{p}\left(x_{0}, B\right)$. Since $a_{i} \in D_{p}\left(x_{0}, B\right)$, by Lemma 5.1 we have

$$
a_{i}=\lambda_{i} x_{0}+\left(1-\lambda_{i}^{p}\right)^{1 / p} b_{i}, \quad \text { where } \quad b_{i} \in B, 0 \leq \lambda_{i} \leq 1 .
$$

Since $\left(\lambda_{i}\right) \subset[0,1]$, there exists a subsequence $\left(\lambda_{i_{j}}\right)$ convergent to some $\lambda_{0} \in$ $[0,1]$.

If $\lambda_{0}=1$, then $\left(1-\lambda_{i_{j}}^{p}\right)^{1 / p} b_{i_{j}} \rightarrow 0$ and $\lambda_{i_{j}} x_{0} \rightarrow \lambda_{0} x_{0}=x_{0}$. Hence $a_{i_{j}}=\lambda_{i_{j}} x_{0}+\left(1-\lambda_{i_{j}}^{p}\right)^{1 / p} b_{i_{j}} \rightarrow x_{0}$. Thus $a_{0}=x_{0} \in D_{p}\left(x_{0}, B\right)$.

If $0 \leq \lambda_{0}<1$, then in $E_{A}$,

$$
\lambda_{i_{j}} x_{0} \rightarrow \lambda_{0} x_{0} \quad \text { and } \quad \lambda_{i_{j}} x_{0}+\left(1-\lambda_{i_{j}}^{p}\right)^{1 / p} b_{i_{j}} \rightarrow a_{0} .
$$

Hence in $E_{A}$,

$$
\left(1-\lambda_{i_{j}}^{p}\right)^{1 / p} b_{i_{j}} \rightarrow a_{0}-\lambda_{0} x_{0} \quad \text { and } \quad b_{i_{j}} \rightarrow \frac{a_{0}-\lambda_{0} x_{0}}{\left(1-\lambda_{0}^{p}\right)^{1 / p}}
$$

Put

$$
b_{0}:=\frac{a_{0}-\lambda_{0} x_{0}}{\left(1-\lambda_{0}^{p}\right)^{1 / p}} .
$$

Then $b_{0} \in B$ since $B$ is locally closed. Thus

$$
a_{0}=\lambda_{0} x_{0}+\left(1-\lambda_{0}^{p}\right)^{1 / p} b_{0} \in D_{p}\left(x_{0}, B\right) .
$$

Lemma 5.4 (see [13, p. 101] or [27, p. 94]). Let $S \subset X$ be an absolutely $p$-convex set. Then for any scalars $\alpha_{1}, \ldots, \alpha_{n}$, we have $\alpha_{1} S+\cdots+\alpha_{n} S \subset \gamma S$ provided that $\gamma \geq\left(\sum_{i=1}^{n}\left|\alpha_{i}\right|^{p}\right)^{1 / p}$.

Proof. Since $\sum_{i=1}^{n}\left|\alpha_{i} / \gamma\right|^{p} \leq 1$, for any $x_{1}, \ldots, x_{n} \in S$, we have $\sum_{i=1}^{n}\left(\alpha_{i} / \gamma\right) x_{i} \in S$. From this, $\sum_{i=1}^{n} \alpha_{i} x_{i} \in \gamma S$, and hence $\alpha_{1} S+\cdots+\alpha_{n} S$ $\subset \gamma S$. 
Lemma 5.5. Let $B \subset X$ be $p$-convex and $A \subset X$ be nonempty. If there exists a p-convex absorbing set $W$ such that $W \cap(A-B)=\emptyset$, then for any $x_{0} \in A$ and any $\varepsilon, 0<\varepsilon<1$, there exists $a \in D_{p}\left(x_{0}, B\right) \cap A$ such that

$$
D_{p}(a, B) \cap A \subset\left\{\lambda a+\left(1-\lambda^{p}\right)^{1 / p} b:(1-\varepsilon)^{1 / p}<\lambda \leq 1, b \in B\right\} .
$$

Proof. Let $q_{W}$ be the gauge of $W$. Since $W \cap(A-B)=\emptyset$ and $W$ is absorbing, we have

$$
1 \leq q_{W}(x-y)<\infty, \quad \forall x \in A, y \in B .
$$

Clearly,

$$
1 \leq \alpha:=\inf \left\{q_{W}(x-y): x \in D_{p}\left(x_{0}, B\right) \cap A, y \in B\right\}<\infty .
$$

For any $\varepsilon$ such that $0<\varepsilon<1$, there exist $a \in D_{p}\left(x_{0}, B\right) \cap A$ and $b_{1} \in B$ such that

$$
q_{W}\left(a-b_{1}\right)<\alpha(1+\varepsilon) .
$$

Take any $z \in D_{p}(a, B) \cap A$. By Lemma 5.1 we may assume that $z=\lambda a+\mu b_{2}$, where $\lambda, \mu \geq 0, \lambda^{p}+\mu^{p}=1$ and $b_{2} \in B$. Since $b_{1}, b_{2} \in B$ and $B$ is $p$-convex, we have

$$
\lambda b_{1}+\mu b_{2} \in B
$$

By Lemma 5.2 we know that $D_{p}(a, B) \subset D_{p}\left(x_{0}, B\right)$, hence

$$
z \in D_{p}\left(x_{0}, B\right) \cap A \text {. }
$$

From (10), (12) and (13), we have

$$
\begin{aligned}
\alpha & \leq q_{W}\left(z-\lambda b_{1}-\mu b_{2}\right)=q_{W}\left(\lambda a+\mu b_{2}-\lambda b_{1}-\mu b_{2}\right) \\
& =q_{W}\left(\lambda\left(a-b_{1}\right)\right)=\lambda^{p} q_{W}\left(a-b_{1}\right) .
\end{aligned}
$$

Now using (11), we obtain

$$
\alpha \leq \lambda^{p} q_{W}\left(a-b_{1}\right)<\lambda^{p} \alpha(1+\varepsilon) .
$$

Since $0<1 \leq \alpha<\infty$, we have $1<\lambda^{p}(1+\varepsilon)$. From this,

$$
1-\varepsilon<\frac{1}{1+\varepsilon}<\lambda^{p}, \quad \text { and so } \quad(1-\varepsilon)^{1 / p}<\lambda .
$$

Thus we have shown that

$$
\begin{aligned}
D_{p}(a, B) \cap A & \subset\left\{\lambda a+\mu b:(1-\varepsilon)^{1 / p}<\lambda \leq 1, \mu \geq 0, \lambda^{p}+\mu^{p}=1, b \in B\right\} \\
& =\left\{\lambda a+\left(1-\lambda^{p}\right)^{1 / p} b:(1-\varepsilon)^{1 / p}<\lambda \leq 1, b \in B\right\} .
\end{aligned}
$$

Now we can give a $p$-drop theorem in locally $p$-convex spaces under a very weak assumption.

TheOrem 5.1. Let $X$ be a locally complete locally $p$-convex space, $A \subset X$ be locally closed and $B \subset X$ be locally closed, bounded and $p$-convex. If there exists a p-convex absorbing set $W$ such that $W \cap(A-B)=\emptyset$, then for any $x_{0} \in A$ there exists $a \in D_{p}\left(x_{0}, B\right) \cap A$ such that $D_{p}(a, B) \cap A=\{a\}$. 
Proof. Take a sequence $\left(\varepsilon_{n}\right)$ of positive real numbers such that

$$
0<\varepsilon_{n}<1-\left(1-1 / 2^{n}\right)^{p}<1 / 2^{n}, \quad n=1,2, \ldots
$$

By Lemma 5.5 there exists $a_{1} \in D_{p}\left(x_{0}, B\right) \cap A$ such that

$$
D_{p}\left(a_{1}, B\right) \cap A \subset\left\{\lambda a_{1}+\left(1-\lambda^{p}\right)^{1 / p} b:\left(1-\varepsilon_{1}\right)^{1 / p}<\lambda \leq 1, b \in B\right\} .
$$

Similarly, there exists $a_{2} \in D_{p}\left(a_{1}, B\right) \cap A$ such that

$$
D_{p}\left(a_{2}, B\right) \cap A \subset\left\{\lambda a_{2}+\left(1-\lambda^{p}\right)^{1 / p} b:\left(1-\varepsilon_{2}\right)^{1 / p}<\lambda \leq 1, b \in B\right\} .
$$

Continuing, we obtain a sequence $\left(a_{n}\right)$ with

$$
\begin{aligned}
a_{1} & =\lambda_{0} x_{0}+\left(1-\lambda_{0}^{p}\right)^{1 / p} b_{0}, \quad \text { where } 0 \leq \lambda_{0} \leq 1, b_{0} \in B, \\
a_{2} & =\lambda_{1} a_{1}+\left(1-\lambda_{1}^{p}\right)^{1 / p} b_{1}, \quad \text { where }\left(1-\varepsilon_{1}\right)^{1 / p}<\lambda_{1} \leq 1, b_{1} \in B, \\
& \vdots \\
a_{n+1} & =\lambda_{n} a_{n}+\left(1-\lambda_{n}^{p}\right)^{1 / p} b_{n}, \quad \text { where }\left(1-\varepsilon_{n}\right)^{1 / p}<\lambda_{n} \leq 1, b_{n} \in B,
\end{aligned}
$$

Hence

$$
\begin{gathered}
a_{n+2}-a_{n+1}=\left(1-\lambda_{n+1}^{p}\right)^{1 / p} b_{n+1}-\left(1-\lambda_{n+1}\right) a_{n+1}, \\
\vdots \\
a_{n+k+1}-a_{n+k}=\left(1-\lambda_{n+k}^{p}\right)^{1 / p} b_{n+k}-\left(1-\lambda_{n+k}\right) a_{n+k} .
\end{gathered}
$$

By adding the above $k$ equalities, we obtain

$$
\begin{aligned}
a_{n+k+1}-a_{n+1}= & \left(1-\lambda_{n+1}^{p}\right)^{1 / p} b_{n+1}+\cdots+\left(1-\lambda_{n+k}^{p}\right)^{1 / p} b_{n+k} \\
& -\left(1-\lambda_{n+1}\right) a_{n+1}-\cdots-\left(1-\lambda_{n+k}\right) a_{n+k} .
\end{aligned}
$$

Obviously for every $i$,

$$
b_{n+i} \in B \subset \Gamma_{p}\left(x_{0}, B\right) .
$$

Also, by Lemma 5.2,

$$
a_{n+i} \in D_{p}\left(a_{n+i-1}, B\right) \subset \cdots \subset D_{p}\left(a_{n}, B\right) \subset \cdots \subset D_{p}\left(x_{0}, B\right) \subset \Gamma_{p}\left(x_{0}, B\right),
$$

where $\Gamma_{p}\left(x_{0}, B\right)$ is circled, hence

$$
-a_{n+i} \in \Gamma_{p}\left(x_{0}, B\right) .
$$

Combining (14)-(16) and applying Lemma 5.4, we have

$$
\begin{aligned}
a_{n+k+1}-a_{n+1} \in & \left(1-\lambda_{n+1}^{p}\right)^{1 / p} \Gamma_{p}\left(x_{0}, B\right)+\cdots+\left(1-\lambda_{n+k}^{p}\right)^{1 / p} \Gamma_{p}\left(x_{0}, B\right) \\
& +\left(1-\lambda_{n+1}\right) \Gamma_{p}\left(x_{0}, B\right)+\cdots+\left(1-\lambda_{n+k}\right) \Gamma_{p}\left(x_{0}, B\right) \\
\subset & {\left[\varepsilon_{n+1}+\cdots+\varepsilon_{n+k}+\left(1-\left(1-\varepsilon_{n+1}\right)^{1 / p}\right)^{p}+\cdots\right.} \\
& \left.+\left(1-\left(1-\varepsilon_{n+k}\right)^{1 / p}\right)^{p}\right]^{1 / p} \Gamma_{p}\left(x_{0}, B\right) .
\end{aligned}
$$


By the choice of $\left(\varepsilon_{n}\right)$ we know that $\left(1-\varepsilon_{n}\right)^{1 / p}>1-1 / 2^{n}$, so $1-\left(1-\varepsilon_{n}\right)^{1 / p}<$ $1 / 2^{n}$ and hence

$$
\left(1-\left(1-\varepsilon_{n}\right)^{1 / p}\right)^{p}<1 / 2^{n p}
$$

so from (17) we have

$$
\begin{aligned}
a_{n+k+1}-a_{n+1} & \in\left[\frac{1}{2^{n+1}}+\cdots+\frac{1}{2^{n+k}}+\frac{1}{2^{(n+1) p}}+\cdots+\frac{1}{2^{(n+k) p}}\right]^{1 / p} \Gamma_{p}\left(x_{0}, B\right) \\
\subset & \left(\frac{1}{2^{n}}+\frac{1}{2^{p}-1} \cdot \frac{1}{2^{n p}}\right) \Gamma_{p}\left(x_{0}, B\right) .
\end{aligned}
$$

Thus $\left(a_{n}\right)$ is a locally Cauchy sequence, and hence is locally convergent to some point $a$. Next we consider two cases.

CASE 1. Assume that there exists a subsequence $\left(a_{n_{i}}\right)_{i \in \mathbb{N}}$ of $\left(a_{n}\right)_{n \in \mathbb{N}}$ such that $a_{n_{i}}=x_{0}$ for all $i$. Then for any $z \in D_{p}\left(x_{0}, B\right) \cap A$, we have $z \in D_{p}\left(a_{n_{i}}, B\right) \cap A$ for $i=1,2, \ldots$, and $z$ can be written as $\left.z=\lambda_{n_{i}}^{\prime} a_{n_{i}}+\left(1-\lambda_{n_{i}}^{\prime}\right)^{p}\right)^{1 / p} b_{i}^{\prime}$, where $\left(1-\varepsilon_{n_{i}}\right)^{1 / p}<\lambda_{n_{i}}^{\prime} \leq 1$ and $b_{i}^{\prime} \in B$. Letting $i \rightarrow \infty$, we obtain $z=\lim _{i \rightarrow \infty}\left(\lambda_{n_{i}}^{\prime} a_{n_{i}}+\left(1-\left(\lambda_{n_{i}}^{\prime}\right)^{p}\right)^{1 / p} b_{i}^{\prime}\right)=\lim _{i \rightarrow \infty}\left(\lambda_{n_{i}}^{\prime} x_{0}+\left(1-\left(\lambda_{n_{i}}^{\prime}\right)^{p}\right)^{1 / p} b_{i}^{\prime}\right)=x_{0}$. Hence $D_{p}\left(x_{0}, B\right) \cap A=\left\{x_{0}\right\}$.

CASE 2. Assume that there exists some $m \in \mathbb{N}$ such that $a_{n} \neq x_{0}$ for every $n>m$. We may assume that $a_{n} \neq x_{0}$ for all $n$. Since $B$ is a locally closed bounded $p$-convex set, by Lemma $5.3, D_{p}\left(a_{n}, B\right)$ is locally closed and hence $D_{p}\left(a_{n}, B\right) \cap A$ is locally closed. As $\left(a_{n+k}\right)_{k \in \mathbb{N}} \subset D_{p}\left(a_{n}, B\right) \cap A$ and $\left(a_{n+k}\right)_{k \in \mathbb{N}}$ is locally convergent to $a$, we infer that $a \in D_{p}\left(a_{n}, B\right) \cap A$. This holds for every $n$, so $a \in \bigcap_{n=1}^{\infty} D_{p}\left(a_{n}, B\right) \cap A$. We assert that $D_{p}(a, B) \cap A$ $=\{a\}$. In fact, take any $z \in D_{p}(a, B) \cap A$. Then $z \in D_{p}\left(a_{n}, B\right) \cap A$ for every $n$. Thus we may assume that

$$
z=\mu_{n} a_{n}+\left(1-\mu_{n}^{p}\right)^{1 / p} y_{n}, \quad \text { where }\left(1-\varepsilon_{n}\right)^{1 / p}<\mu_{n} \leq 1, y_{n} \in B .
$$

Hence

$$
\begin{aligned}
z-a_{n} & =\left(1-\mu_{n}^{p}\right)^{1 / p} y_{n}+\mu_{n} a_{n}-a_{n} \\
& \in\left(1-\mu_{n}^{p}\right)^{1 / p} \Gamma_{p}\left(x_{0}, B\right)+\left(1-\mu_{n}\right) \Gamma_{p}\left(x_{0}, B\right) \\
& \subset\left[1-\mu_{n}^{p}+\left(1-\mu_{n}\right)^{p}\right]^{1 / p} \Gamma_{p}\left(x_{0}, B\right) \\
& \subset\left[\varepsilon_{n}+\left(1-\left(1-\varepsilon_{n}\right)^{1 / p}\right)^{p}\right]^{1 / p} \Gamma_{p}\left(x_{0}, B\right) \\
& \subset\left(\frac{1}{2^{n}}+\frac{1}{2^{n p}}\right)^{1 / p} \Gamma_{p}\left(x_{0}, B\right) \subset\left(\frac{1}{2^{n p}}+\frac{1}{2^{n p}}\right)^{1 / p} \Gamma_{p}\left(x_{0}, B\right) \\
& \subset \frac{2^{1 / p}}{2^{n}} \Gamma_{p}\left(x_{0}, B\right) .
\end{aligned}
$$


Thus $\left(a_{n}\right)$ is locally convergent to $z$. At the same time, it is locally convergent to $a$. Therefore $z=a$, i.e., $D_{p}(a, B) \cap A=\{a\}$.

REMARK 5.1. From the proof of Theorem 5.1 we observe that every $a_{n} \in D_{p}\left(x_{0}, B\right) \cap A$. If $A$ or $B$ is locally complete, we can deduce that $D_{p}\left(x_{0}, B\right) \cap A$ is still locally complete. Thus we see that in Theorem 5.1 the condition that $X$ is locally complete can be replaced by the condition that $B$ or $A$ is locally complete.

\section{References}

[1] J.-P. Aubin and I. Ekeland, Applied Nonlinear Analysis, Wiley, New York, 1984.

[2] J. Caristi, Fixed point theorems for mappings satisfying inwardness conditions, Trans. Amer. Math. Soc. 215 (1976), 241-251.

[3] L. Cheng, Y. Zhou and F. Zhang, Daneš' drop theorem in locally convex spaces, Proc. Amer. Math. Soc. 124 (1996), 3699-3072.

[4] J. Daneš, A geometric theorem useful in nonlinear functional analysis, Boll. Un. Mat. Ital. 6 (1972), 369-375.

[5] -, Equivalence of some geometric and related results in nonlinear functional analysis, Comment. Math. Univ. Carolin. 26 (1985), 443-454.

[6] I. Ekeland, Sur les problèmes variationnels, C. R. Acad. Sci. Paris Sér. A 275 (1972), $1057-1059$.

[7] —, On some variational principle, J. Math. Anal. Appl. 47 (1974), 324-354.

[8] —, Nonconvex minimization problems, Bull. Amer. Math. Soc. 1 (1979), 443-474.

[9] J. X. Fang, The variational principle and fixed point theorems in certain topological spaces, J. Math. Anal. Appl. 202 (1996), 398-412.

[10] P. G. Georgiev, The strong Ekeland variational principle, the strong drop theorem and applications, ibid. 131 (1988), 1-21.

[11] A. H. Hamel, Phelps' lemma, Daneš' drop theorem and Ekeland's principle in locally convex spaces, Proc. Amer. Math. Soc. 131 (2003), 3025-3038.

[12] J. Horváth, Topological Vector Spaces and Distributions, Vol. 1, Addison-Wesley, Reading, MA, 1966.

[13] H. Jarchow, Locally Convex Spaces, Teubner, Stuttgart, 1981.

[14] G. Köthe, Topological Vector Spaces I, Springer, Berlin, 1983.

[15] D. Kutzarova and C. Leránoz, On $p$-drop property, $0<p<1$, Atti Sem. Mat. Fis. Uni. Modena 42 (1994), 89-102.

[16] M. Landsberg, Lineare topologische Räume, die nicht lokalkonvex sind, Math. Z. 65 (1956), 104-112.

[17] N. Mizoguchi, A generalization of Brøndsted's result and its application, Proc. Amer. Math. Soc. 108 (1990), 707-714.

[18] D. Pallaschke and S. Rolewicz, Foundations of Mathematical Optimization, Math. Appl. 388, Kluwer, Dordrecht, 1997.

[19] J. P. Penot, The drop theorem, the petal theorem and Ekeland's variational principle, Nonlinear Anal. 10 (1986), 813-822.

[20] P. Pérez Carreras and J. Bonet, Barrelled Locally Convex Spaces, North-Holland, Amsterdam, 1987.

[21] R. R. Phelps, Convex Functions, Monotone Operators and Differentiability, Springer, Berlin, 1989. 
[22] J. H. Qiu, Local completeness and drop theorem, J. Math. Anal. Appl. 266 (2002), 288-297.

[23] —, Ekeland's variational principle in locally complete spaces, Math. Nachr. 257 (2003), 55-58.

[24] - Local completeness, drop theorem and Ekeland's variational principle, J. Math. Anal. Appl. 311 (2005), 23-39.

[25] J. H. Qiu and S. Rolewicz, Local completeness of locally pseudoconvex spaces and Borwein-Preiss variational principle, Studia Math. 183 (2007), 99-115.

[26] S. Rolewicz, On a certain class of linear metric spaces, Bull. Acad. Polon. Sci. 5 (1957), 471-473.

[27] —, Metric Linear Spaces, PWN-Polish Sci. Publ., Warszawa, and Reidel, Dordrecht, 1985.

[28] X. Y. Zheng, Drop theorem in topological vector spaces, Chinese Ann. Math. Ser. A 21 (2000), 141-148 (in Chinese).

Department of Mathematics

Institute of Mathematics

Suzhou University

Suzhou, Jiangsu 215006

Polish Academy of Sciences

People's Republic of China

E-mail: jhqiu@suda.edu.cn

P.O. Box 21, Śniadeckich 8

00-956 Warszawa, Poland

E-mail: rolewicz@impan.gov.pl

Received September 15, 2006

Revised version February 21, 2008 